\title{
Estucoqueratosis. A propósito de un caso
}

\author{
Stucco keratosis. A case report
}

\section{Eva Rubal Lamelas ${ }^{1}$, Benigno Monteagudo Sánchez², Aquilina Ramírez Santos ${ }^{3}$, Juan Carlos Álvarez Fernández ${ }^{4}$, Abián Mosquera Fernández ${ }^{5}$}

\author{
${ }^{1}$ Grado en Podología. Universidade da Coruña \\ evinharl@gmail.com \\ ${ }^{2}$ Licenciado en Medicina y Cirugía. Servicio de Dermatología. Complejo Hospitalario Universitario de Ferrol. Área Sani- \\ taria de Ferrol-Servicio Gallego de Salud. \\ benigno.monteagudo.sanchez@sergas.es \\ ${ }^{3}$ Licenciado en Medicina y Cirugía. Servicio de Dermatología. Complejo Hospitalario Universitario de Santiago-Servicio \\ Gallego de Salud. \\ aquilina.ramirez.santos@sergas.es \\ ${ }^{4}$ Licenciado en Medicina y Cirugía. Jefe de Servicio de Anatomía Patológica. Complejo Hospitalario Universitario de \\ Ferrol. Área Sanitaria de Ferrol-Servicio Gallego de Salud. \\ juan.carlos.alvarez.fernandez@sergas.es \\ ${ }^{5}$ Profesor contratado doctor. Departamento de Ciencias de la Salud. Facultad de Enfermería y Podología. Universidade da \\ Coruña, Campus de Ferrol. \\ abian@udc.es
}

\section{Correspondencia:}

Dr. Benigno Monteagudo Sánchez

Servicio de Dermatologia - Hospital Naval de Ferrol

Avda. de la Residencia $\mathrm{s} / \mathrm{n}$

E-15405 Ferrol (A Coruña)

Correo-e: benigno.monteagudo.sanchez@sergas.es

Fecha de recepción: 30 de septiembre de 2015

Fecha de aceptación: 4 de octubre de 2015

Los autores declaran no tener ningún tipo de interés económico o comercial.

\section{RESUMEN}

La estucoqueratosis es una patología dérmica que cursa con tumoraciones queratósicas asintomáticas, benignas, blanco-grisáceas y de pequeño tamaño. Éstas suelen localizarse en las extremidades (especialmente en las inferiores) en torno al tobillo. Su etiología es desconocida y su diagnóstico se realiza mediante una correcta anamnesis y exploración física ya que la morfología, localización y edad de presentación son claves para poder establecer un diagnóstico diferencial con otras afecciones aunque en caso necesario también se puede recurrir a la biopsia. Constituye una entidad clínica con especial interés podológico dada su frecuente aparición en las extremidades inferiores, de ahí la necesidad de conocerla y de saber realizar un correcto diagnóstico diferencial. Presentamos el caso de un varón de 45 años sin antecedentes dermatológicos que presenta estucoqueratosis en la extremidad inferior y que acude al Servicio de Dermatología del Hospital Naval de Ferrol.

Palabras clave: acantosis; estucoqueratosis; papilomatosis; pápulas; verrugas planas.

\section{ABSTRACT}

Stucco keratosis is a dermal pathology that causes small, white-greyish, asymptomatic benign keratotic tumors. These are usually located in the extremities (especially in the lower) around the ankle. Its etiology is unknown and diagnosis is made through a proper clinical history and physical examination as morphology, location and age of presentation are key to establishing a diferential diagnosis with other conditions although if necessary biopsy is also avalaible. Stucco keratosis is a clinical entity with special podiatric interest given its frequent appearance in the lower extremities, hence the need to know and be able to perform a correct differential diagnosis. We report the case of a 45 year old man with no previous dermatological history presenting stucco keratosis in the lower extremity admitted to the outpatient clinic in the Dermatology Department of the Naval Hospital of Ferrol.

Key words: acanthosis; stucco keratosis; papillomatosis; papules; flat warts.

Referencia normalizada: Rubal Lamelas. E., Monteagudo Sánchez, B., Ramírez Santos, A., Álvarez Fernández, J.C., Mosquera Fernández, A. Estucoqueratosis. A propósito de un caso. Rev. Int. Cienc. Podol. 2016; 10(1): 26.30.

Sumario: 1. Introducción. 2. Caso clínico. 3. Discusión. Bibliografía. 


\section{INTRODUCCIÓN}

Descrita por primera vez por Kocsar y Ofner en $1965^{1}$ la estucoqueratosis constituye un proceso benigno y poco frecuente caracterizado por la aparición de pequeñas pápulas queratósicas, normalmente asintomáticas, de color blancogrisáceo que suelen localizarse en las extremidades superiores e inferiores (principalmente en tobillos). El número de lesiones puede variar desde unas pocas a cientos de ellas y no se ha descrito degeneración maligna. Algunos autores la consideran un tipo de queratosis seborreica debido a que su histopatología es similar a la de la queratosis seborreica hiperqueratósica ${ }^{2-3}$.

\section{CASO CLÍNICO}

Varón de 45 años, con antecedentes personales de hipercolesterolemia, amigdalectomía y cirugía por fractura de codo izquierdo que acude a consulta por la presencia de lesiones pruriginosas en el dorso de pies y de manos de 7 años

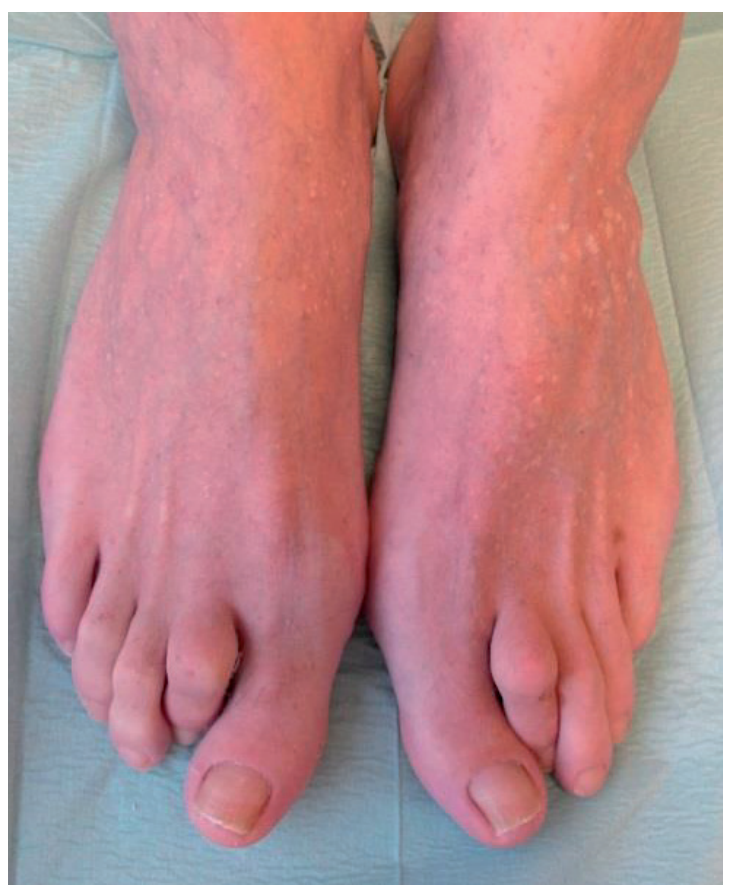

Figura. 1. Pápulas queratósicas de diámetro inferior a $2 \mathrm{~mm}$ en el dorso de ambos pies.

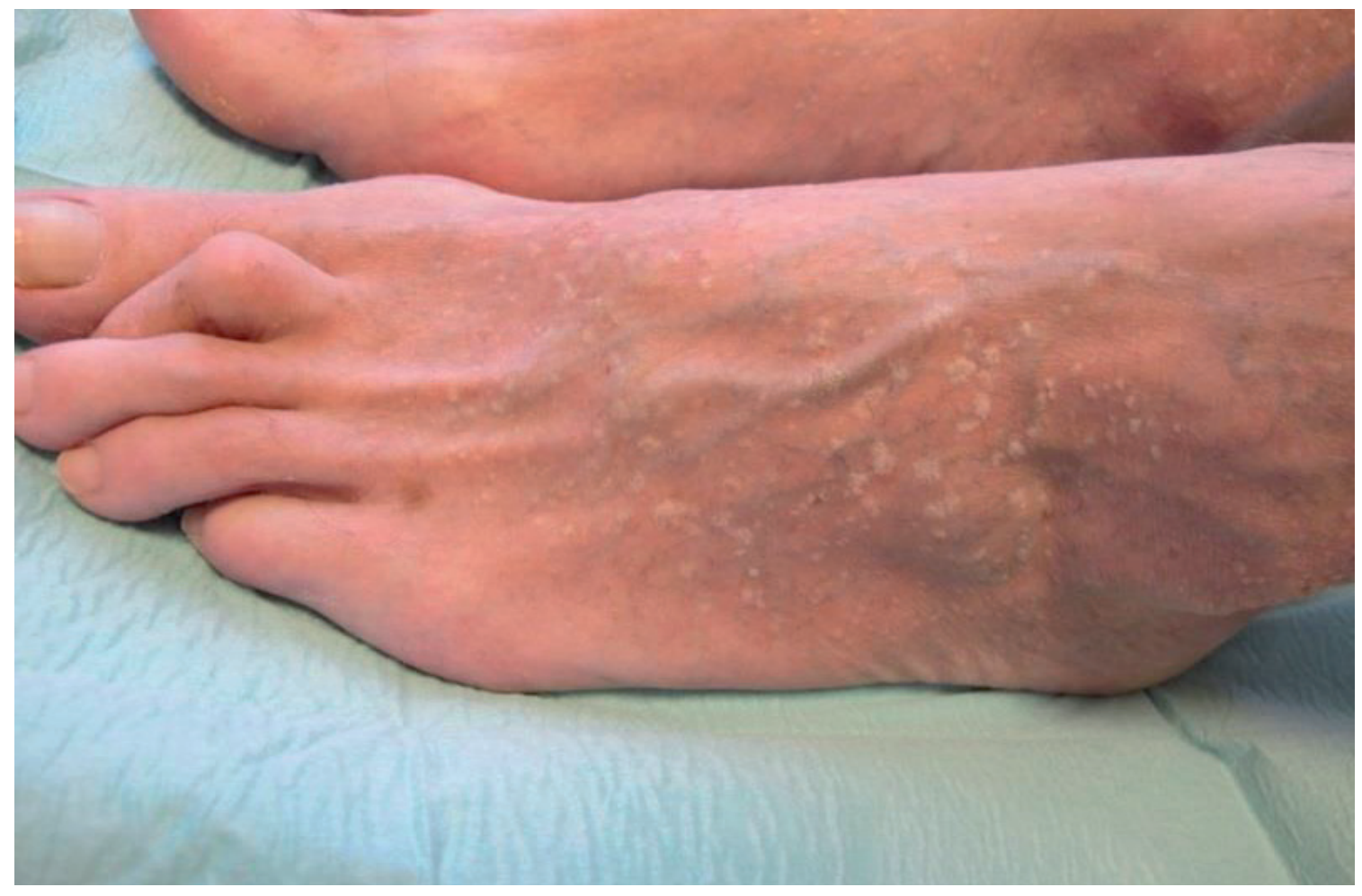

Figura 2. Detalle de las lesiones. 


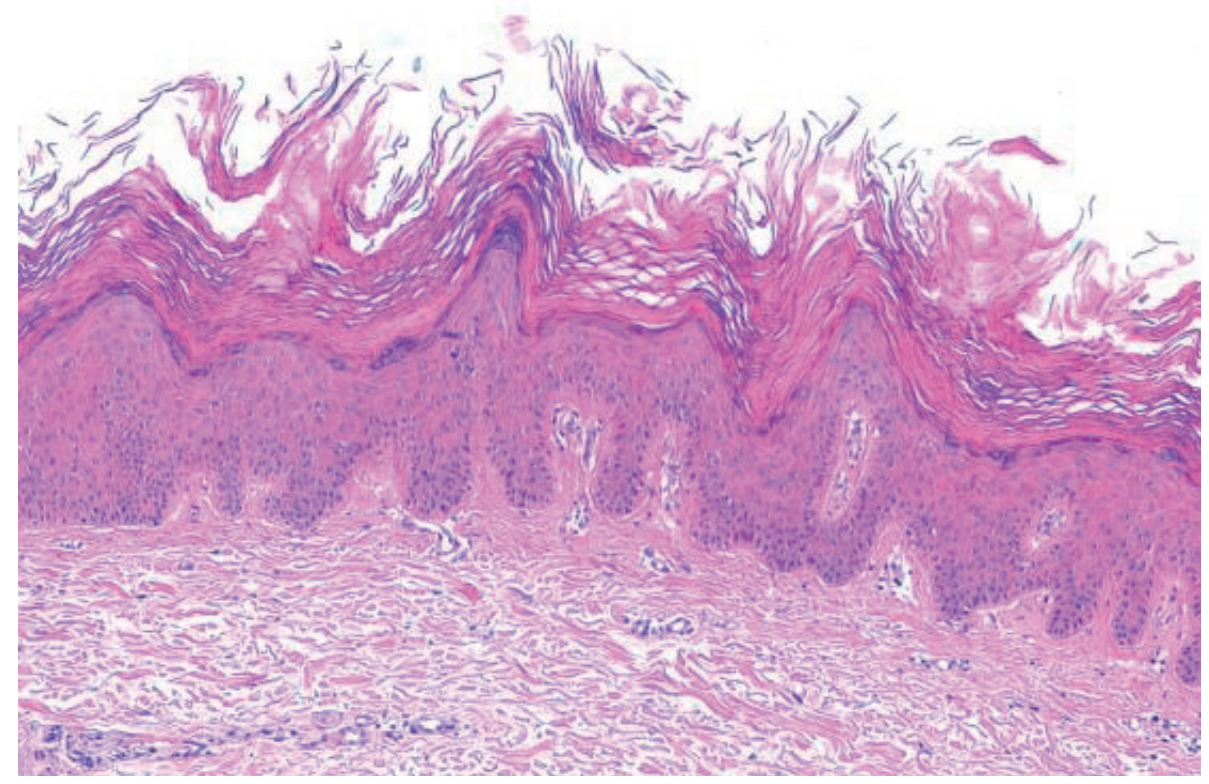

Figura 3. Papilomatosis con acantosis e hiperqueratosis ortoqueratósica (hematoxilina-eosina, $\times 100$ ).

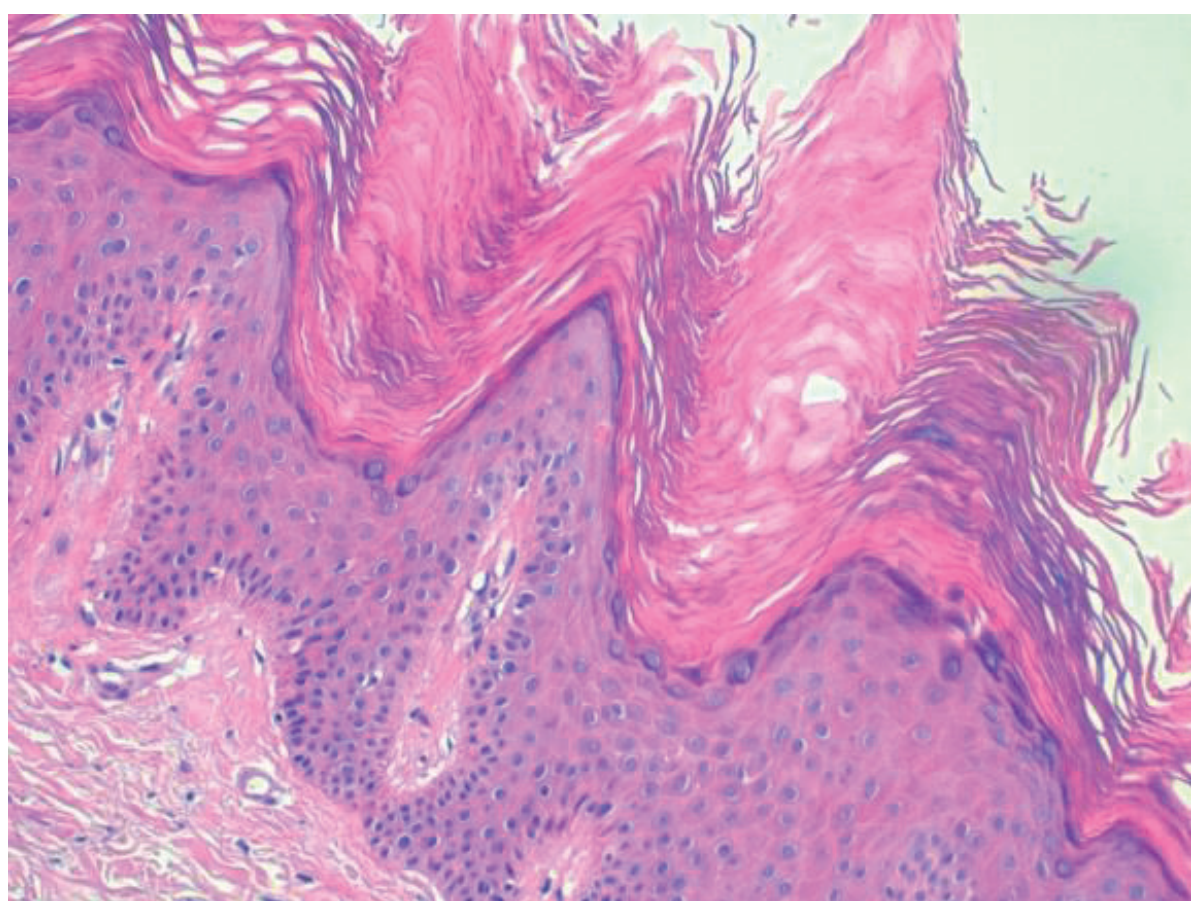

Figura 4. Acúmulos de queratina "en campanario de iglesia" (hematoxilina-eosina, ×200). 
de evolución que paulatinamente han aumentado en número causando prurito con el calor. A la exploración se observan múltiples pápulas queratósicas blanquecinas de menos de $2 \mathrm{~mm}$ de diámetro, bien delimitadas, localizadas sobre todo en el dorso de ambos pies y, de forma aislada, en extremidades superiores (Figuras 1 y 2).

Para su estudio histológico se llevó a cabo la exéresis de una de las lesiones que mostró acantosis de patrón especulado en la epidermis y una marcada hiperqueratosis ortoqueratósica y papilomatosis sobre una dermis sin cambios significativos (Figuras 3 y 4). Con el diagnóstico de estucoqueratosis se pautó tratamiento tópico con vaselina salicilada al 5\%.

\section{DISCUSIÓN}

La estucoqueratosis es una entidad más frecuente en varones de edad avanzada (proporción hombre/mujer 4:1). Su morfología clínica, localización y edad de presentación son algunas de las claves que permiten diferenciarla de otras patologías como pueden ser la queratosis seborreica, la epidermodisplasia verruciforme, la queratosis actínica, la hiperqueratosis lenticularis perstans, la acroqueratoelastoidosis de Costa, la enfermedad de Darier, la acroqueratosis verruciforme de Hopf, el nevo epidérmico verrugoso, la poroqueratosis actínica superficial diseminada y las verrugas planas ${ }^{3-6}$. En el ámbito podológico tiene especial interés el diagnóstico diferencial entre la estucoqueratosis y las verrugas planas. A diferencia de la estucoqueratosis las verrugas planas suelen localizarse en el área facial e histológicamente se caracterizan por ortoqueratosis con paraqueratosis y coilocitosis ${ }^{3,7}$. Debido a su naturaleza benigna y tendencia a la involución espontánea, la estucoqueratosis no suele tratarse salvo por razones estéticas. Para su tratamiento se ha empleado entre otros el ácido salicílico, retinoico, tricloroacético, la electrocoagulación, el curetaje, el 5 fluoruracilo, el maxacalcitol, el imiquimod y el etretinato.

En este caso concreto al paciente se le pautó vaselina salicilada al 5\%, compuesto que con frecuencia se ha empleado para el tratamiento de la hiperqueratosis. Su utilidad radica en que a concentraciones superiores al 1\%-2\% actúa como queratolítico provocando la eliminación parcial de la capa córnea de la epidermis consiguiendo así disminuir su espesor y eliminar esas pequeñas pápulas que aparecen de manera inherente a la patología ${ }^{8-10}$. El nitrógeno líquido constituye otra alternativa terapéutica aunque, debido al limitado número de lesiones que pueden ser tratadas en cada sesión para evitar quemaduras extensas, no es una terapia de rápida resolución que además de resultar dolorosa por lo general presenta frecuentes recidivas ${ }^{11}$.

En otros casos de estucoqueratosis se ha descrito la aplicación de imiquimod en crema al 5\% 3 veces a la semana durante 5 semanas. Además existen terapias cuyo objetivo no es eliminar las lesiones sino controlar los síntomas. En este sentido para controlar el prurito puede ser necesaria la aplicación de una crema emoliente tras el aseo diario ${ }^{12}$.

Para concluir consideramos necesario que el clínico sepa reconocer la entidad clínica conocida como estucoqueratosis y diferenciarla de otros procesos dermatológicos como las queratosis actínicas o las verrugas planas para de esa manera evitar el empleo de tratamientos innecesarios y contraindicados que no resolverán la patología.

\section{BIBLIOGRAFÍA}

1. Kocsard E, Carter JJ. The papillomatous keratoses. The nature and differential diagnosis of stucco keratosis. Australas J Dermatol. 1971; 12(2):80-88.

2. Thomas VD, Swanson NA, Lee KK. Benign epitelial tumors, hamartomas, and hiperplasias. En: Fitzpatrick T. Dermatología en medicina general. $7^{\mathrm{a} e d}$. Madrid: Panamericana; 2009:1054- 1067.

3. Sábat M, Ribera M. Pápulas hiperqueratósicas en las piernas. Piel. 2001; 16(2):107-109.

4. Díaz T, Mateu A. Pápulas queratósicas en áreas acrales. Piel. 2010; 25(7):397-398.

5. Díaz T, Mateu A. Pápulas queratósicas en áreas acrales. Diagnóstico y comentario. Piel. 2010; 25(7):401-402.

6. Ontón J, Cortez F, Carayhua D, Rengifo L. Pápulas verrucosas en extremidades y tronco superior. 
Dermatol Peru. 2006; 16(3):256-260.

7. Chavanne U, Carbia S, Etchart C, La Forgia M. Pápulas asintomáticas en miembros superiores. Dermatol Argent. 2009; 15 (5):368-371.

8. Malpassi LS, Dancziger E, Zeitlin E. Pápulas hiperqueratósicas en miembros inferiores. Dermatol Argent. 2007; 13(3):210-213.

9. Teraki Y, Sato Y, Izaki S. Successful treatment of stucco keratosis with maxacalcitol. Br J Dermatol. 2006; 155(5):1085-1086.

10. Grupo de trabajo de Acofarma. Fichas de información técnica: ácido salicílico.Disponible en: http:// www.acofarma.com/admin/uploads/descarga/4334-de72bf4c49b7949a54289c0caaa894af1336d511/ main/files/cido_salic_lico.pdf.

11. Monroe J. Multiple "warts" on the lower legs of a 52-years-old woman. JAAPA. 2005; 18(11):66.

12. Stockfleth E, Rowert J, Arndt R, Christophers E, Meyer T. Detection of human papillomavirus and response to topical 5\% Imiquimod in a case of stucco keratosis. Br J Dermatol. 2000; 143(4): 846-850. 